\title{
Padecimientos emocionales, búsqueda de ayuda y expectativas de atención en una comunidad urbano-marginal
}

Jazmín Mora-Ríos, D ra en Psic, ${ }^{(1)}$ Ma Emily Ito-Sugiyama, Dra en Psic. ${ }^{(2)}$

\section{Mora-Ríos J, Ito-Sugiyama ME. \\ Padecimientos emocionales, \\ búsqueda de ayuda y expectativas de atención en una comunidad urbano-marginal. Salud Publica Mex 2005;47:145-154.}

\section{Resumen}

Objetivo. C onocer la experiencia de los habitantes de una comunidad urbano marginal respecto de sus padecimientos emocionales, a quiénes recurren para enfrentarlos y sus expectativas de atención. Material y métodos. La información que se analiza en este trabajo se basa en una aproximación multimetodológica. El abordaje teórico inició en el año 2000, en la D elegación Magdalena Contreras, del Distrito Federal, y la recolección de la información se hizo de 2001 a 2003. El análisis cualitativo fue simultáneo al levantamiento de las entrevistas en profundidad (16), durante el periodo 2000-2002. En el primer semestre de este último año se aplicaron los cuestionarios (204) en población adulta - hombres y mujeres-, y en el segundo semestre se capturó la información. El análisis cuantitativo se hizo en 2003. Resultados. La falta de una red de apoyo social es una condición que comparten los informantes, el apoyo esperado para hacer frente a los padecimientos emocionales es, para las mujeres, la familia nuclear; aunque en la práctica la pareja suele ser más una fuente de malestar. En el caso de los hombres, el apoyo esperado proviene primordialmente de la pareja. Conclusiones. Se plantea una serie de reflexiones en torno a la intervención, considerando la participación de los habitantes de la comunidad y el trabajo interdisciplinario.

Palabras clave: padecimientos emocionales; salud mental; comunidad; búsqueda de ayuda; expectativas de atención; México

\author{
Mora-Ríos J, Ito-Sugiyama ME. \\ Emotional ailments, \\ help-seeking behaviors and care expectancies \\ in a marginal-urban community. \\ Salud Publica Mex 2005;47:145-154.
}

\begin{abstract}
A bstract
Objective.To obtain information about emotional ailments based on the experience of adults in a socially underserved urban community, their social support resources to cope with them, and their health care expectancies. Material and Methods. The study began in 2000, in the Magdalena Contreras D istrict of Mexico C ity. D ata collection was conducted in 2001-2003, using a multimethodological approach that included in depth interviews of female and male adults ( $n=16)$ performed during 2000-2002. A semistructured questionnaire $(n=204)$ was applied in the first semester of 2002 and data entering was performed in the second semester of that year. Q uantitative analysis was performed in 2003. Results The community informants evidenced a lack of social support networks. For women, the expected support to cope with emotional disorders should come from the nuclear family, although in practice their partner represented a so urce of discontent. For men, the main expected source of support was their wives. Conclusions. Based on these results, some reflections are proposed around intervention programs considering both the perspective of the community members and the multidisciplinary work.
\end{abstract}

Key words: emotional ailments; mental health; community; help seeking processes; health care expectancies; Mexico

(1) Instituto Nacional de Psiquiatría Ramón de la Fuente, (IN PRF). D irección de Investigaciones Epidemiológicas y Sociales. México, DF, México.

(2) Universidad N acional Autónoma de México, Facultad de Psicología. México, DF, México.

Fecha de recibido: 2 de junio de 2004 - Fecha de aprobado: 2 de febrero de 2005

Solicitud de sobretiros: Dra. Jazmín Mora-Ríos. D epartamento de Investigaciones Psicosociales. Dirección de Investigaciones Epidemiológicas y Sociales. Avenida México-X ochimilco, N 0. 101 colonia San Lorenzo Huipulco. 14370. México, DF.

Correo electrónico: morarj@imp.edu.mx. 
E n las últimas dos décadas se ha observado un creciente interés en el campo de la investigación en salud mental, lo que ha estimulado la generación de conocimientos nuevos sobre el amplio espectro de trastornos mentales. Hoy en día, el interés se enfoca en las poblaciones más vulnerables que viven en condiciones de marginación y pobreza en países como Africa, Asia y Latinoamérica, gracias al reconocimiento de que la salud mental es un componente central de los problemas de salud derivados de la desigualdad que caracteriza a los países en desarrollo. ${ }^{1}$

La disparidad en los niveles de salud entre las clases sociales no desaparece por la igualdad en el acceso a los servicios públicos. La salud se relaciona con las condiciones y estilos de vida, por lo que las oportunidades de mantener una forma de vida saludable siguen diferenciando a las clases sociales. Estas oportunidades están limitadas, entre otras razones, por factores como el deterioro en las condiciones de vida, vivienda precaria, dieta pobre o inadecuada, menores ingresos, alcoholismo, abuso de drogas, la oferta de servicios y la disponibilidad de apoyos comunitarios. Así, el estilo de vida que promueve una vida saludable aparece como más típico de las clases medias y altas. ${ }^{2}$

Para algunos autores como Boltansky, ${ }^{3}$ la desigualdad social en el campo de la atención a la salud no sólo se refleja en la mayor presencia de enfermedad, sino que, además, el acceso a los recursos y las respuestas institucionales plasman estas divergencias sociales. En el caso de México, por ejemplo, existe una distribución desigual de los servicios de salud a consecuencia de la concentración de recursos en las principales ciudades, y una desatención grave en ciertas áreas pobres del país, aunado al hecho de que una gran mayoría de la población no cuenta con los servicios de seguridad social por parte del Estado.

Desde una perspectiva de salud pública, la importancia de brindar atención a la salud mental radica no sólo en el alto costo económico que representan para México padecimientos como la esquizofrenia y la depresión, que ocupan el segundo lugar en importancia en el campo de la salud, ${ }^{4}$ sino que los índices de mortalidad revelan que la dependencia al alcohol es el principal contribuyente en el exceso de riesgo en perder un año de vida saludable. También resultan significativas las pérdidas ocasionadas por los episodios depresivos, así como la problemática de la violencia: la tasa de homicidios es casi tres veces mayor que el promedio mundial por 1000 habitantes. ${ }^{5}$

De acuerdo con la Encuesta Nacional de Epidemiología Psiquiátrica en México, solamente 1 de cada 10 que padece algún trastorno mental recibe atención ${ }^{6}$ y son diversas las razones que explican esta situación.
En principio, son muy escasos los servicios de atención en salud mental y, cuando los hay, prácticamente no se recurre a ellos ya sea por el desconocimiento de la población acerca de los padecimientos emocionales, o bien porque los profesionales de la salud no satisfacen las expectativas de atención.

El interés por conocer cómo se vive el proceso salud enfermedad, en el caso específico de la salud mental, en el contexto de una comunidad que vive en condiciones de pobreza, de ningún modo se basa en el supuesto de que la patología se concentra en estos sectores, en comparación con otros grupos con ingresos más elevados, sino que la vulnerabilidad está dada en términos de las desigualdades en la atención a la salud, ya que se ha encontrado que las mayores carencias que padece la población de escasos recursos económicos es la relacionada con la salud y la seguridad social, ${ }^{7}$ y es justamente en la salud mental donde se refleja esta inequidad, pues constituye una de las áreas más desatendidas en México. Esto se explica por el rezago que existe en los campos de investigación, atención y prevención, aspectos que han sido documentados en la literatura. $^{8}$

Después del fracaso de los programas de intervención, que se elaboran sin considerar las necesidades de las poblaciones a quienes supuestamente van dirigidos, hoy en día el interés radica en la importancia de recuperar la experiencia y los contextos locales en diferentes países de Latinoamérica empleando estrategias metodológicas de carácter cualitativo que permitan involucrar a los participantes en la toma de decisiones. ${ }^{9}$

La importancia que tiene el conocer las trayectorias de la búsqueda de ayuda frente a los padecimientos emocionales que se derivan de las situaciones problemáticas de la vida diaria estriba en considerar los recursos formales e informales para enfrentar estas situaciones. Esta información resulta crucial para el desarrollo de programas sociales en las comunidades, pues se propone que deben incorporar la visión de los destinatarios de dichos programas con el fin de garantizar su eficacia.

Las orientaciones en salud que hacen un abordaje parcial y fragmentado de los aspectos físicos y emocionales pueden constituirse en barreras para el tratamiento, como el enfocarse exclusivamente en los aspectos físicos de la salud por considerar que los emocionales no son prioritarios en los sectores más desfavorecidos de la población, aun cuando existen estudios que dan cuenta de su importancia tanto en población rural $^{10}$ como urbana. ${ }^{11}$

El objetivo de este trabajo consiste en identificar los padecimientos percibidos por la población adulta en el contexto de una comunidad marginal, y conocer 
la relación que guardan los factores emocionales con la salud de los participantes. Adicionalmente, se analizan los recursos de apoyo frente a los padecimientos emocionales y las expectativas de los participantes en torno a los programas de atención en esta área.

El marco teórico de referencia fue la teoría de las representaciones sociales, ${ }^{12}$ que cuenta con amplia tradición en el campo de la salud mental y permite acceder al conocimiento de los significados que atribuyen los actores sociales a la salud y la enfermedad mental. El origen de esta teoría se encuentra en el supuesto de que existe una serie de normas socioculturales a partir de las cuales las personas desarrollan creencias e interpretaciones acerca de la naturaleza de la sociedad, de sí mismas y de las relaciones sociales, y que las percepciones de sus estados emocionales estarán mediadas por la naturaleza de esas creencias. Para una revisión de la teoría de las representaciones sociales se recomienda la lectura de los textos clásicos de Serge Moscovici ${ }^{13}$ y Denise Jodelet. ${ }^{14}$ En este documento se adoptó una aproximación de carácter más procesual, ${ }^{15}$ utilizando una perspectiva multimetodológica ${ }^{16}$ con objeto de destacar el carácter dinámico de la teoría y desarrollar estrategias de investigación para enriquecer la gama de aproximaciones a nuestros objetos de estudio en el ámbito de la salud mental.

\section{Material y métodos}

La investigación se desarrolló entre 2001 y 2003, en una comunidad del sur de la Ciudad de México, Distrito Federal elegida por ser la de mayor nivel de marginalidad en esta demarcación. * Se trata de un área de asentamientos irregulares que carece de todos los servicios. No obstante, existen actividades comunitarias denominadas "faenas", de carácter obligatorio, en las cuales participan los habitantes de la comunidad para proveerse de algunos de estos servicios. La población es heterogénea no sólo en cuanto al lugar de origen, pues la mayor parte proviene de diversos estados de la República Mexicana como Oaxaca, Querétaro, Estado de México y Puebla, e incluso de otros países como Honduras y Guatemala; también lo es en términos religiosos y políticos. En ese sentido, se trata de una comunidad de paso, ya que muchas familias permanecen

\footnotetext{
* Gobierno de la Ciudad de México-Secretaría de Salud. La marginación socioeconómica de los hogares del Distrito Federal. Programa de Educación para la Salud de la Familia; 2000 (disco compacto).
}

ahí por un tiempo, y luego prosiguen su migración hacia los Estados Unidos de América en busca de mejores oportunidades laborales.

Este trabajo forma parte de un estudio más amplio que incluyó entrevistas a diversos informantes, observaciones de tipo etnográfico y la aplicación de un cuestionario semiestructurado para caracterizar a la población en términos sociodemográficos y de salud que aparecen descritos detalladamente en trabajos previos. ${ }^{17,11}$ Aquí, únicamente se consideró la información del cuestionario de salud ( $n=204)$ y de las entrevistas en profundidad con la población adulta de la comunidad $(n=16)$. Estas últimas, para lograr un mayor acercamiento e ilustrar las experiencias de los habitantes de la comunidad en relación con sus padecimientos emocionales y la búsqueda de ayuda.

\section{Entrevistas en profundidad}

Se diseñó una guía de entrevista que contenía diferentes tópicos de estudio. En este trabajo se presenta la información relacionada con los padecimientos reportados por los entrevistados, las atribuciones a los mismos y la búsqueda de ayuda para enfrentarlos.

El contacto con la población adulta de la comunidad se estableció a través de los trabajadores de la salud que en ese momento brindaban atención a los habitantes de la comunidad. Posteriormente, se empleó la técnica "bola de nieve". El procedimiento general para recabar la información consistió en la colaboración voluntaria del entrevistado a participar en un estudio cuya finalidad era conocer las opiniones sobre aspectos de salud en la comunidad, y se ofreció una breve explicación de sus objetivos. Durante la trascripción de las entrevistas, los nombres de los informantes, grupos, instituciones, e incidentes que facilitaran su identificación, fueron cambiados y en su lugar se utilizaron pseudónimos.

\section{Cuestionario de salud}

El cuestionario fue aplicado de forma directa por el entrevistador, quien además de llevar el registro por escrito de las respuestas de los informantes, anotaba sus observaciones alrededor de aspectos significativos para el estudio. Cada aplicación duró entre 25 y 30 minutos.

A todos los participantes se les explicó que el manejo de la información era estrictamente confidencial y sería utilizada únicamente con fines de estudio. Asimismo, y a solicitud de los informantes, se brindaron opciones de atención incluso entre quienes no acepta- 
ron participar en la investigación, de manera que fueron remitidos a los centros de atención gratuita más cercanos a la comunidad. El proyecto de investigación fue aprobado por el Comité de Bioética del Instituto Nacional de Psiquiatría "Ramón de la Fuente".

Para el análisis cualitativo de la información proveniente de las entrevistas se hicieron dos tipos de análisis: temático y textual. Para el temático se adoptaron los procedimientos sugeridos por González-Martínez, ${ }_{1}^{18}$ los cuales se basan en la identificación de categorías y subcategorías de análisis generadas a partir de los tópicos de estudio contenidos en la guía de entrevista. Respecto del análisis textual se utilizó un programa especializado: Alceste (versión 4.5)..$^{19,20}$ Se trata de un método de análisis de textos que se inscribe en la tradición del desarrollo de técnicas estadísticas aplicadas al análisis de datos lingüísticos que permite la exploración del discurso libre de los sujetos y cuyo procedimiento se describe en un trabajo previo. ${ }^{11}$ Para la información derivada del cuestionario se obtuvieron frecuencias simples a las preguntas abiertas a través del programa estadístico SPSS (versión 10.1).

\section{Resultados}

La información sociodemográfica se muestra en el cuadro I. En relación con el empleo la mayor parte de los habitantes son trabajadores eventuales; en el caso de las mujeres el trabajo doméstico, el comercio ambulante y ser ayudantas de albañil fueron las principales actividades. Asimismo, también hacen uso de la vivienda para incrementar los ingresos familiares, ya sea vendiendo diversos artículos (dulces, cosméticos, alcohol, alimentos o fritangas) o desempeñando el trabajo a destajo al interior de sus hogares en horarios que permitan combinar estas actividades con la crianza de los hijos y las labores domésticas. En el caso de los hombres predominan las actividades relacionadas con la construcción, los servicios, los negocios por cuenta propia y, en menor medida, los empleados públicos y privados. El ingreso semanal familiar promedio corresponde a 600 pesos \pm 407 , cantidad con la que viven cinco personas, es decir, cada persona vive al día, en promedio, con 17 pesos. El salario mínimo correspondiente a 2001 y 2003, fecha en que se hizo el trabajo de campo, osciló entre los 35 y 38 pesos, respectivamente, ingresos insuficientes para satisfacer las necesidades mínimas básicas. De los informantes encuestados $(n=204)$, tan sólo $15 \%$ cuenta con un sistema de seguridad social y el resto de la población tiene que recurrir a diferentes opciones en busca de respuestas efectivas a sus padecimientos.

\section{Cuadro I \\ Características sociodemográficas de los PARTICIPANTES EN EL ESTUDIO SOBRE PADECIMIENTOS EMOCIONALES EN UNA COMUNIDAD URBANO MARGINAL. MÉxıco, DF, 2001-2003}

$\frac{\text { Mujeres }(n=132)}{\text { frecuencia } \%} \frac{\text { Varones }(n=72)}{\text { frecuencia } \%}$

Edad

\begin{tabular}{rrrrr}
\hline $22-27$ años & 16 & 12.1 & 4 & 5.6 \\
\hline $28-33$ años & 40 & 30.3 & 25 & 34.7 \\
\hline $34-39$ años & 40 & 30.3 & 14 & 19.4 \\
\hline $40-45$ años & 28 & 21.1 & 19 & 26.4 \\
\hline $46-51$ años & 8 & 6.2 & 10 & 13.9
\end{tabular}

Estado civil

\begin{tabular}{lrrrr} 
Con pareja & 125 & 94 & 69 & 96 \\
\hline Sin pareja & 7 & 6 & 3 & 4
\end{tabular}

Empleo

\begin{tabular}{lrrrr} 
Con trabajo remunerado & 55 & 42 & 67 & 93 \\
\hline Sin trabajo remunerado & 77 & 58 & 5 & 7
\end{tabular}

$n=204$

\section{Padecimientos reportados por el informante en el último año}

Mediante una pregunta abierta del cuestionario se indagó acerca de los padecimientos en el último año, así como las atribuciones que hicieron los informantes sobre los mismos. Como se observa en el cuadro II, las infecciones en las vías respiratorias (52\%) (por ejemplo gripe, amigdalitis, dolor de garganta, tos, neumonía, bronquitis y sinusitis) fueron el principal padecimiento mencionado, y se atribuyó al clima frío que predomina en la zona. Aun cuando sólo 9\% de los entrevistados refirieron padecimientos relacionados con el estrés, lo que incluye tanto malestar emocional como manifestaciones somáticas, estos ocuparon el segundo lugar, entre otros destacan: "embolia por los corajes", "caída de pelo por preocupaciones", "colitis nerviosa por una sorpresa y preocupaciones", "úlcera por estrés", "nervios por problemas de trabajo", "parálisis facial por un coraje", "presión alta por las preocupaciones y los nervios", "presión baja por enojos", "hipertensión por estrés", "falta de memoria por las presiones", "vesícula por corajes", "presión alta por mucha tensión", "nervios por problemas con la pareja", "presión alta 


\section{Cuadro II \\ Padecimientos en el último año, reportados POR LOS PARTICIPANTES EN EL ESTUDIO SOBRE PADECIMIENTOS EMOCIONALES EN UNA COMUNIDAD urbano-marginal. México, DF, 2001-2003}

\begin{tabular}{lrr} 
& Frecuencia & $\%$ \\
Infecciones respiratorias & 105 & 52 \\
\hline Padecimientos atribuidos al estrés & 19 & 9 \\
\hline Infecciones gastrointestinales & 13 & 6 \\
\hline Padecimientos relacionados con el corazón & 8 & 4 \\
\hline Accidentes & 6 & 3 \\
\hline Dolores musculares & 5 & 2 \\
\hline Padecimientos sexuales & 4 & 2 \\
\hline Afecciones en el pulmón & 3 & 1 \\
\hline Alergias & 2 & 1 \\
\hline Otros* & 8 & 5 \\
\hline Ninguna & 30 & 15 \\
$*$ Asma, varicela, diabetes, anemia, hepatitis, dermatitis, hernias, afecciones \\
del riñón \\
$\begin{array}{l}\text { \% de no respuesta=2 (1\%) } \\
\text { (n=204) }\end{array}$
\end{tabular}

por tensiones y preocupaciones", "migraña", "nervios por problemas familiares y de trabajo", "no come por las preocupaciones"). En lo relativo a los dolores musculares, los informantes mencionaron diversas partes del cuerpo como la cabeza, la espalda, dolor de huesos, muchos de los cuales se atribuyeron al exceso de trabajo.

Los padecimientos gastrointestinales (diarrea, dolor de estómago), ocuparon el tercer lugar (6\%). En relación con los padecimientos sexuales en las mujeres, éstas mencionaron los "quistes en los ovarios", "ulceraciones en la matriz", "infecciones y hemorragias vaginales". En el caso de los hombres, se mencionaron los accidentes de trabajo, por ejemplo lesiones y cortaduras en brazos y las caídas. Sólo 15\% notificó no haber tenido ningún padecimiento en el último año.

Conforme a la información obtenida de las entrevistas en profundidad, los padecimientos respiratorios afectan a la población en general. En los niños destacó el asma, epilepsia, problemas de aprendizaje y de lenguaje, además del abuso sexual en los hijos de dos entrevistados por parte de un familiar cercano, y el consumo de drogas. Para el caso de los hombres se mencionaron las enfermedades de transmisión sexual (por ejemplo infecciones), y los accidentes principalmente referidos a la actividad laboral. $\mathrm{Al}$ respecto, conviene recordar que la actividad preponderante es la del trabajador de la construcción; por lo tanto, los riesgos de trabajo (caídas, golpes, raspones, quemaduras), al igual que el consumo de alcohol, están muy ligados a esta actividad. Las mujeres, hablaron ampliamente sobre su salud sin establecer distinciones entre los padecimientos físicos y emocionales, como se observa en el siguiente relato:

"[...] y sí, esta parte me estaba sintiendo bien mal, como que me mareo, como que me he estado sintiendo como débil, como que me quiero caer...Yo creo que es porque no me alimento bien, porque hago corajes, este, por la presión muy alta que se me sube por lo que, luego me da este...Me duele mucho la cabeza y sí, sí, como que me pongo..., pero antes porque no me alimentaba bien, pero orita ya me alimento más o menos con lo que gano [...] me dan poco pero saco, saco las tortillas ¿no?, pero donde estaba antes, este, con un tal señor Edmundo, no comía o sea le decía yo no comía, un día que me pasa de comer porque este, hicimos todo el escombro y medio comía, tons, quedé...débil de lo que no comía muy bien yo, entonces, orita ya...Ya se me están controlando un poco los mareos pero sí, sí este, ya no me mareo tanto" (Elena, 29 años).

\section{Búsqueda de ayuda frente a los padecimientos emocionales}

En el cuadro III se muestran las respuestas a la pregunta del cuestionario "¿a quién recurre cuando se siente triste o deprimido?". A partir de estas respuestas se observa que los recursos de apoyo esperados frente a los padecimientos emocionales son muy variados y evidencian la consideración de múltiples recursos para enfrentarlos. La principal fuente de apoyo proviene de la familia nuclear; básicamente se trata de la pareja y los hijos. Otro recurso importante está representado por los miembros de la familia de origen (padres, hermanos), familiares cercanos y otras figuras significativas como los compadres, amigas(os), tíos, cuñados y patrones, entre otros. Algunos incluyen a la espiritualidad (Dios). El 17\% de los participantes prefiere hacer frente por sí mismo a sus padecimientos. Sin embargo, al considerar la información obtenida de las entrevistas en profundidad, se hace evidente que en la práctica la disponibilidad de estos recursos es limitada, como se muestra a continuación.

Si bien la familia de origen es una fuente de apoyo para las mujeres, principalmente las madres y hermanos, la lejanía geográfica dificulta recurrir a este recurso: 


\section{Cuadro III \\ Personas a quienes recurren cuando SE SIENTEN TRISTES O DEPRIMIDOS LOS PARTICIPANTES EN EL ESTUDIO SOBRE PADECIMIENTOS EMOCIONALES EN UNA COMUNIDAD URBANO-MARGINAL. MÉxıco, DF, 2001-2003}

\begin{tabular}{lrrrrrrr} 
& \multicolumn{2}{c}{ Mujeres } & \multicolumn{2}{c}{ Hombres } & \multicolumn{2}{c}{ Total } \\
& frecuenciá & $\%$ & frecuencia & $\%$ & frecuencia & $\%$ \\
El o ella sola(o) & 21 & 16 & 13 & 18 & 34 & 17 \\
\hline Pareja & 19 & 14 & 30 & 43 & 49 & 24 \\
\hline A migos & 13 & 10 & 3 & 4 & 16 & 8 \\
\hline Hijos & 14 & 11 & 1 & 1 & 7 & 3 \\
\hline Hermano(a) & 7 & 5 & 1 & 1 & 8 & 4 \\
\hline Dios & 6 & 4 & 1 & 1 & 7 & 3 \\
\hline Mamá & 7 & 5 & 1 & 1 & 8 & 4 \\
\hline Comadre/compadre & 3 & 2 & 1 & 1 & 4 & 2 \\
\hline Pareja/hijos & 4 & 3 & 4 & 6 & 8 & 4 \\
\hline Pareja/padres & 3 & 2 & 3 & 4 & 6 & 3 \\
\hline Ella sola y su pareja & 1 & 1 & 2 & 3 & 2 & 1 \\
\hline Padre & - & - & 2 & 3 & 2 & 1 \\
\hline Suegra & 3 & 2 & - & - & 3 & 1 \\
\hline Patrona/patrón & 2 & 1 & - & - & 2 & 1 \\
\hline Cuñada & 2 & 1 & - & - & 2 & 1 \\
\hline Tía & 2 & 1 & - & - & 2 & 1 \\
\hline Amigos/pareja & - & - & 2 & 3 & 2 & 1 \\
\hline Familia & - & - & 2 & 3 & 2 & 1 \\
\hline O tros: & $25 *$ & 19 & $4{ }^{\ddagger}$ & 5 & 29 & 14 \\
\hline N o respuesta & - & - & 2 & 3 & 2 & 1 \\
\hline Total & 132 & 100 & 72 & 100 & 204 & 100 \\
* Por lo menos una mención (Dios-pareja, Dios-madrina, ella sola y sus \\
hermanos, padre, vecina y madre, sacerdote, pareja/hermano, hermana/ \\
tía, concuña, D ios, ella sola- hijos, ambos padres, radio)
\end{tabular}

“[...] pus aquí no contamos con ningún familiar, ora sí que se puede decir estamos solos, le digo que él es de Comitán, Chiapas, y su única hermana que tiene está hasta Canadá y pues la verdad ni él ni yo contamos con nadie, entonces, este, no le digo ora sí que lo que se dice amistad, pues un amigo de él, pero todos sus amigos como que no, no hay uno que le diga, que hable con él, que le haga ver las cosas, que le diga: 'reflexiona', al contrario, le meten cosas o le dan alcohol, entonces no, en ese sentido no" (Yolanda, 33 años).

Al mismo tiempo, en el caso de las mujeres, la pareja aparece como una de las principales fuentes de malestar. Las entrevistadas se refirieron ampliamente a las expectativas de apoyo, no cubiertas, por parte de la pareja en situaciones difíciles, en las cuales la salud de las mujeres se ve involucrada:

"Haciendo el quehacer y cuando tengo que salir todo el día casi no tengo tiempo a descansar ni estar sentada. Porque ni enferma me estoy sentada, ando para allá y para acá, pues porque no puedo, una vez estaba yo bien enferma, bien enferma y me fui caminando hasta donde estaba el doctor que sí me atendía bien, con el que me llevó la señora, como a las 12 de la noche, porque no encontraba la medicina en ningún lado, no hay esas medicinas, sólo con el doctor... en su... en su.... dispensario... Y así me fui en la noche y mi marido bien dormido que ni cuenta se dio. Pero ese doctor sí me revivió. Me ayudó mucho su medicina" (Magdalena, 42 años).

Institucionalmente, las experiencias de los entrevistados son muy variadas, sin embargo, predominaron aquellas que hablan de las expectativas de apoyo no cubiertas por parte de los profesionales de la salud. A continuación se incluye un relato que ejemplifica lo anterior:

"[...] fui a pedir asesoramiento allá al DIF y todo para ver si me daban terapia o algo así, entonces me dijo no que... Ah, pero para esto, fui con él y me dijeron que no, que ahí que no estaban para dividir matrimonios sino para unirlos y la psicóloga que me trató me dijo que lo que querían es que ya no querían matrimonios rotos, que ya no los querían y que por eso había tantísimos problemas en la sociedad y le digo pero es que yo ya no aguanto, yo ya no quiero estar con él. No, dice, perdónalo mira que [...]" (Patricia, 34 años).

El autocuidado es otra estrategia que emplean las mujeres en un afán por sentirse mejor. Por ejemplo, "tratar de descansar", "tomar el sol un rato", "darse ánimo a sí misma", "tratar de no estar sola" y "hacer ejercicio", el "tratar de controlar sus nervios" y "ser fuerte", como se observa en los siguientes relatos:

“[...] mire, yo tengo un carácter muy voluble pero no sé, a la vez sé controlarme mucho, y así yo veo las cosas tengo que calmarme, pero...no sé, este trato, ¿no? de calmarme y llevar e ignorar lo que veo y lo que oigo porque sé hasta dónde soy capaz de llegar y luego me dicen -bueno, ¿cómo es que nunca se enoja?"- porque siempre ando riendo, si la señora no me habla pues ni modo, aunque tal vez le caiga mal a alguien siempre se controla uno" (Yolanda, 33 años). 
Por otra parte, el permanecer encerrado en la casa "para evitar problemas" constituye una norma no escrita que los hombres imponen a sus parejas. Por ejemplo, cuando se preguntó a un entrevistado acerca de personas cercanas o allegadas a su esposa respondió lo siguiente:

"[...] lo que pasa es que ella no es de problemas, ella nada más se dedica a su casa y sus hijos, pues más que nada aquí porque aquí es pura gente que de una cosa inventa bastante más, la verdad, y ella no es de esas personas, mejor se encierra en su casa o se va a ver a sus niños" (Juan, 32 años).

Las implicaciones que se derivan de transgredir esta norma pueden ser muy serias para las mujeres, como se observa en el siguiente relato, en el que la violencia en contra de una mujer la ejerce su pareja por el simple hecho de platicar y contar su vida a otras personas. Así lo expresa uno de los entrevistados al referirse a las razones para abusar de su pareja.

"Le pegaba porque, este, es muy conflictiva, le gusta mucho platicar con las gentes y contar su vida. Entonces en una de esas que regresó [...] traté de pegarle pero no en el estómago sino de enseñarla a base de golpes, entonces ella tenía por ahí un machete, agarra el machete, señorita, y se me echa encima, yo me defendí, no le miento aquí tengo la huella de los dos machetazos que me aventó, de acá esta huella no me alcanzó a dar, pero de los dos machetazos se los esquivé, me defendí con una mano, entonces ya en el tercer machetazo, le contrarresto (sic.) su ataque entonces ella decía: -'mi panza, mi panza' en cuanto le contrarrestré (sic) su ataque se cae y empieza a decir: -'mi panza, mi panza' ya sangrando se recuperó y se fue a vivir con una señora que vive allí, al lado [...]" (Gregorio, 58 años).

Aunque en el caso de los hombres fue más difícil conseguir entrevistas, los recursos que ellos utilizan para buscar "alivio" o cura ante sus malestares es recurrir muy a menudo al consumo de alcohol para liberarse de los problemas cotidianos como de los problemas económicos, la falta de empleo o los de pareja. En este punto coincidieron las mujeres entrevistadas al referirse a los malestares de los hombres. Otra de las posibles razones por las cuales se dice que el consumo es muy elevado es porque el alcohol es un factor que a los varones de esta comunidad les confiere identidad y los provee de cierto grado de seguridad pues no sólo refuerza la cohesión en el grupo sino que es parte de la economía de las redes en estos sectores, ya que se crean los contactos con los patrones y con otros trabajadores para ampliar las opciones de empleo, incluso al interior de la misma.

La falta de una red de apoyo social es una condición que comparten todos los habitantes de la comunidad, lo cual se refleja en los abundantes relatos en donde los vecinos, la comunidad y hasta algunos miembros de la familia fueron percibidos de manera negativa por los entrevistados. En cuanto a los vecinos, pareciera haber un discurso socialmente compartido de desconfianza hacia el otro, pues los entrevistados, tanto mujeres como hombres, lo asocian con problemas, pleitos chismes. Sin embargo, al considerar las prácticas sociales durante las observaciones en la comunidad, se encontró que frecuentemente se establecen redes de solidaridad para obtener ayuda de diversa índole, como los préstamos en dinero y en especie, cuidado de la vivienda y de los hijos, por mencionar unos ejemplos.

Dios fue mencionado frecuentemente a lo largo de las entrevistas; se percibe como una entidad que protege en momentos de adversidad, que provee de los cuidados y de salud a la familia, y representa una alternativa significativa para enfrentar las situaciones de crisis (económica, de vivienda y de salud).

\section{Expectativas de atención}

Desde el punto de vista de los participantes, se requiere de una serie de medidas para mejorar la salud de los habitantes. En primer lugar, la dotación de servicios básicos (53\%), que incluye la regularización de vivien$\mathrm{da}$, escuelas cercanas, espacios recreativos para los niños, como deportivos y parques con juegos, transporte público al interior de la comunidad y mejorar los servicios con los que ya cuentan.

En segundo lugar, mencionaron la implantación de programas de atención en la comunidad (36\%), que van desde la continuidad de los programas gubernamentales de tipo asistencial, que de hecho siguen existiendo desde hace varios años (dotación de despensas, ayudas con material de construcción, útiles, distribución de algunos alimentos, etcétera). Sin embargo, durante las entrevistas individuales y grupales hubo un acuerdo generalizado de que estos programas han tenido un impacto negativo, pues si bien había un efecto positivo inmediato, también fomentaba "la flojera" en muchas familias y la apatía de la comunidad, lo que se reflejó en los siguientes comentarios: "Lo que sí, ojalá y borraran ya todo ese tipo de despensas, porque aquí no nos llega ni siquiera", "es negocio pero para la persona que lo recibe", "las personas que las traen la usan 
como política". Además de que lo asociaron con la corrupción de líderes y autoridades de la delegación, por ser el recurso más utilizado por los políticos para ganar adeptos.

Asimismo, manifestaron su interés por que se llevaran a cabo "pláticas de orientación" sobre diversos temas, como sexualidad, planificación familiar, "orientación a los jóvenes para comunicarse y alejarse de los vicios","orientación a los padres para no maltratar a los hijos", "cursos donde nos enseñen cómo ser cariñosas con los niños", "pláticas de cómo van creciendo los niños", "programas para evitar el maltrato a las mujeres", "que nos enseñen cómo enfrentar problemas pues hay mucha flojera entre la gente", programas dirigidos a combatir las adicciones y el consumo de alcohol entre los jóvenes y los hombres de la comunidad, cursos impartidos por un nutriólogo donde les enseñen a cocinar los alimentos con higiene y con poco dinero, atención psicológica hacia la población en general, cursos dirigidos a la familia "porque falta comprensión y entendimiento en la familia" y para que "no se desintegren tan fácilmente", talleres sobre valores "que les enseñen a los niños que los principios son fundamentales". Para los entrevistados varones fue fundamental un programa dirigido al empleo, donde se brinden apoyos con el fin de conseguir trabajo, así como desarrollar talleres sobre manualidades (corte y confección, belleza) destinados a las mujeres y actividades recreativas para la población "para despejar la mente" y talleres para "los hombres que tienen problemas de pareja".

Otras opiniones acerca de las acciones dirigidas en beneficio de la salud de los habitantes de la comunidad fue a través del mejoramiento de los servicios médicos y de salud (35\%), lo que refleja las necesidades de la población de estudio en cuanto a los servicios que actualmente ofrece el centro de salud de la comunidad. Se señaló que les gustaría se brindara atención médica continua, que mejorara el trato hacia la gente, el personal cumpliera con su horario y hubiera dotación de medicamentos a su alcance. También solicitaron cursos de educación para la salud mediante los cuales puedan aprender "primeros auxilios", "qué hacer en caso de que se tengan enfermedades graves" y cursos preventivos: "pláticas para no enfermarse".

Por último, bajo la categoría "trabajar en comunidad" (3.4\%), los entrevistados destacaron otras acciones dirigidas a las expectativas por trabajar conjuntamente con los vecinos para mejorar sus condiciones de vida. Por ejemplo, "ayudarse entre todos", "organización para trabajar", "unirse y entre todos trabajar para mejorar", "un buen líder" y "que la gente quiera cooperar".

\section{Discusión}

Los resultados de esta investigación muestran coincidencias con lo encontrado en población rural ${ }^{10}$ en donde la salud mental, lejos de constituir un factor secundario frente a otras problemáticas de salud en la población que vive en condiciones de pobreza, es parte integral de la salud y debiera considerarse en los programas de intervención dirigidos a poblaciones vulnerables. ${ }^{1,21}$

Entre las problemáticas que aquejan a la población están el desarraigo, en términos de la pérdida de la cultura de referencia y la consecuente pérdida de redes sociales de ayuda y de sostén, así como la escasez de opciones recreativas y de uso del tiempo libre. Estos factores podrían explicar los padecimientos más referidos por la comunidad, como el consumo de alcohol, la violencia intrafamiliar, las adicciones y la depresión que, en la mayoría de los casos, no están siendo atendidos.

Como se observa al abordar la salud mental, es ineludible hacer referencia a las variables macroestructurales como la pobreza y el género. En esta investigación, los entrevistados aluden al contexto socioeconómico como fuente de malestar, de manera que la satisfacción de necesidades básicas constituye un componente importante de la salud mental. La población de estudio es un grupo vulnerable por las precarias condiciones desde las cuales desempeñan sus actividades laborales en tiempos de incertidumbre económica, que se ubican principalmente dentro del sector informal, sin prestaciones de ninguna especie y de carácter eventual en la mayoría de los casos. Estas condiciones tienen implicaciones en la salud pues en este ámbito se reflejan las desigualdades de lo que para los entrevistados representa el "estar enfermo, sin dinero y sin ir al doctor".

La familia es el recurso más esperado para hacer frente a los padecimientos emocionales; para los entrevistados el principal apoyo es la pareja, en tanto que para las mujeres los recursos de apoyo se orientan hacia la familia de origen (madre, hermanos) además de la pareja y los hijos. No obstante, al analizar los relatos de las mujeres entrevistadas se observa que la pareja es a menudo fuente de malestar y que cuentan con apoyos limitados de la familia de origen. Por ello, otro recurso disponible es el autocuidado, que en población rural aparece como el principal tipo de apoyo. ${ }^{10}$

Aun cuando los resultados de investigación en diferentes comunidades durante la década de los sesenta sugieren que la familia es el principal recurso de supervivencia de las familias urbanas marginales, ${ }^{22}$ 
a partir de la década de los ochenta las investigaciones refieren que el debilitamiento de las redes sociales parece ser una constante en estos grupos y es una tendencia que se observa a escala nacional ${ }^{23,24}$ como en diversos países latinoamericanos ${ }^{25, *}$ donde la desconfianza es el modo básico de relación en los sectores empobrecidos. ${ }^{24}$

La experiencia obtenida de esta investigación muestra esta misma tendencia. A pesar de que los entrevistados siguen pensando que la familia representa una fuente de apoyo en momentos críticos, saben de antemano que en la práctica no cuentan con este recurso. Cabe recordar que los entrevistados viven en una periferia de la ciudad y gran parte proviene de diferentes estados de la República en donde se quedan sus familias de origen. Incluso, entre las personas cuyas familias viven en el Distrito Federal, la lejanía de la comunidad hace que los entrevistados no esperen, en casos de urgencia, un apoyo real de la familia.

En suma, tal y como se ha documentado en anteriores trabajos, ${ }^{26}$ se observó una necesidad de apoyo tanto formal como informal. Institucionalmente, es importante destacar que, a diferencia de lo dicho acerca de que la población de los sectores de escasos recursos económicos no acude a los servicios por ignorancia o falta de información, la experiencia obtenida muestra que la población sí acude a los servicios de atención; sin embargo, éstos no brindan una respuesta satisfactoria a sus demandas.

Respecto de las limitaciones del estudio, la principal tiene que ver con la dificultad para incorporar a los participantes de sexo masculino, lo que impidió explorar en profundidad las condiciones que vulneran la salud de los varones en la comunidad. No obstante, se identificaron algunas situaciones que tienen implicaciones para su salud emocional (consumo de alcohol, conflictos de pareja, crisis de identidad como proveedor exclusivo del hogar, crianza de los hijos) y se encuentran ligadas a su condición de género.

\section{Conclusiones}

En términos económicos, religiosos y políticos, plantear una estrategia de intervención para una comunidad tan heterogénea en un momento en que prevalece la desesperanza entre los habitantes propiciada por el manejo inadecuado tanto de las autoridades, partidos políticos y los líderes de la comunidad, parece una ta-

\footnotetext{
* Programa de las Naciones Unidas para el Desarrollo Humano en Chile. Las paradojas de la Modernización. Chile; 1998. Documento interno de trabajo.
}

rea sumamente ardua. ¿Cómo llegar a establecer un punto de acuerdo entre los habitantes, entre quienes siguen demandando programas asistenciales y que "alguien venga a organizar y a poner orden aquí", y aquellos que se inclinan más por la organización interna de los habitantes, que son la minoría? Justamente, el sector de la población de estudio representa el escenario de lo que se vive a principios del siglo XXI en muchos sectores urbanos populares en México y representa un reto para quienes se interesan en el campo de la psicología comunitaria.

En ese sentido, el presente trabajo constituye un primer punto de partida para desarrollar un programa de intervención basado en las necesidades de los habitantes de la comunidad, desde el supuesto de pensar la realidad como una organización compleja y considerando que un problema definido por los integrantes es el mejor camino de acceso para la promoción de la autogestión. ${ }^{25}$ Visto así, resulta importante promover la participación activa de las comunidades en acciones dirigidas a mejorar sus condiciones de salud. La iniciativa es escasa entre los habitantes y esto se refleja en la demanda de programas de carácter asistencial, lo que obstaculiza las posibilidades de cambio o mejoramiento de la salud.

Es conveniente considerar las diferencias de género en cualquier estrategia de intervención, emprendiendo acciones orientadas a mejorar la calidad de vida, e incorporando las necesidades diferenciales de atención en materia de salud y bienestar de mujeres y hombres desde una concepción integral. En el caso de las mujeres, procurar atención a los padecimientos emocionales sin descuidar otros factores que vulneran su bienestar, como la discriminación, la violencia, el hostigamiento sexual, mejorando sus condiciones laborales $\mathrm{y}$, sobre todo, brindándoles protección jurídica.

Aunque los estudios sobre masculinidades se han interesado en las condiciones que vulneran la salud de los varones, el gran reto sigue siendo profundizar en las experiencias de malestar emocional, pues como señala Izquierdo, "si no conocemos las narraciones del sufrimiento de los hombres desde el patriarcado, ¿cómo podemos lograr transformaciones?, por eso es necesario contar con otras representaciones de las mujeres y los hombres que nos sitúen como sujetos sociales capaces de transformar la realidad, más que como objetos".*

En cuanto a las sugerencias para lograr mejoras en los servicios de atención destaca el brindar un abor-

\footnotetext{
* Izquierdo MJ. La sustancia social de la vida emocional. México, DF: Universidad Nacional Autónoma de México, Programa Universitario de Estudios de Género (PUEG); 2004. Documento no publicado.
} 
daje de carácter más integral a la salud sin establecer una separación entre lo físico y lo mental, como se puede evidenciar en los resultados de trabajo a través de los relatos de los entrevistados, que dan cuenta de la manera como los aspectos físicos y emocionales se encuentran ligados, y que se suscitan en un contexto sociocultural más amplio que los profesionales de la salud suelen dejar al margen, lo cual les impide otorgar una respuesta efectiva a las necesidades de la población. Por otra parte, es necesario desarrollar programas que fomenten la autogestión en las comunidades y mejorar la calidad de la atención capacitando a los trabajadores de la salud para lograr el cambio de actitudes a través de un contacto más humano y respetuoso hacia la población que recibe estos servicios.

Una propuesta valiosa, que se deriva de los hallazgos, es considerar a la salud mental como un campo prioritario de la salud pública en poblaciones vulnerables pues, tradicionalmente, se asume que la atención a los padecimientos infecto-contagiosos es más relevante. En esa misma línea, conviene resaltar el carácter interdisciplinario del trabajo en comunidad, ya que el proceso de salud-enfermedad trasciende las fronteras de cualquier disciplina como la sociología, la antropología y la psicología, y cada una, desde su campo, puede hacer importantes contribuciones en términos de intervención.

\section{Referencias}

1. Patel V. Poverty, inequality and mental health in developing countries. En: Leon D,W alt G, ed. Poverty, inequality and health, an international perspective. 0 xford: 0 xford University Press; 2001.

2. Basabe M. Hacia un modelo explicativo del malestar psicológico y somático: alienación y factores psicosociales (tesis doctoral). D eusto: Universidad de Deusto; 1991.

3. Boltansky L. Los usos sociales del cuerpo. Buenos Aires,Argentina: Periferia; 1975.

4. Arredondo A, Ramos R, Zúñiga A. Evaluación económica de la demanda de atención médica para salud mental en México: esquizofrenia y depresión, 1996-2000. Rev Invest Clin 2003:55:43-50. 5. Frenk J, Londoño J, Lozano R. Pluralismo estructurado, una visión para el futuro de los sistemas de salud en América Latina. En: Bronfman M, Castro R, ed. Salud, cambio social y política. Perspectivas desde A mérica Latina. México, D F: Edamex/Instituto N acional de Salud Pública; 1999. 6. Medina-Mora ME, Borges G, Lara C, Benjet C, Blanco J, Fleiz C et al. Prevalencia de trastornos mentales y uso de servicios. Resultados de la Encuesta $N$ acional de Epidemiología Psiquiátrica en México. Salud Ment 2003;26:1-16.
7. Boltvinik J, Hernández-Laos E. Pobreza y distribución del ingreso en México. México, DF: Siglo XXI; 1999.

8. Lara MA, Salgado-de Snyder N . Mujer, pobreza y salud mental. En: Grupo Interdisciplinario sobre Mujer Trabajo y Pobreza (GIMTRAP). Las mujeres en la pobreza. México, DF: El Colegio de México; 1993: 243 291.

9. Cohen A, Kleinman A, Saraceno B.W orld mental health casebook. Social and mental health programs in low income countries. NuevaYork: Kluwer Academic/Plenum Publishers; 2002.

10. Salgado-de Snyder N, Díaz-Pérez MJ, G onzález-Vázquez T. Modelo de integración de recursos para la atención de la salud mental en la población rural de México. Salud Publica Mex 2003;45:19-26.

11. Mora-Ríos J, Flores F, D e Alba M, Marroquín M. Construcción social de significados acerca de la salud mental en una comunidad urbana marginal. Salud Ment 2003;26:51-60.

12. Moscovici S. La psychoanalyse: Son image et son public (D euxiéme édition : 1976). Paris: Presses Universitaires de France; 1961.

13. Moscovici S. El psicoanálisis, su imagen y su público. Buenos Aires: Ed. Huemul; 1979.

14. Jodelet D. La representación social: fenómenos, conceptos y teoría. En: Moscovici S. Psicología Social II. Pensamiento y vida social. Barcelona: Paidós; 1984.

15. Banchs M.A proximaciones procesuales y estructurales al estudio de las representaciones sociales. Papers Soc Representations 1999;8:1-15. 16. De Rosa A. Per un approccio multi-metodo allo studio delle rappresentazioni sociali. Rassi Psicologia 1990;7:101-193.

17. Mora-Ríos J. Una aproximación multimetodológica al estudio de las representaciones sociales de la salud mental en una comunidad urbana marginal. (tesis doctoral). México, DF: U niversidad N acional Autónoma de México; 2004.

18. González-Martínez L. La sistematización y el análisis de los datos cualitativos. En: Mejía-Arauz R, Sandoval SA, ed.Tras las vetas de la investigación cualitativa. Perspectivas y acercamientos desde la práctica. México, DF: ITESO ; 1999.

19. Reinert M. Un logiciel d'analyse lexicale [ALCESTE]. C ah Analyse Données, 1986;471-484.

20. Reinert M.Q Quelques interrogations à propos de l'objet d'une analyse de discours de type statistique et de la réponse Alceste. Lang Société 1999:90:57-85.

21. Desjarlais R, Eisenberg L, Good B, Kleinman A. Salud mental en el mundo. Problemas y prioridades en poblaciones de bajos ingresos. W ashington, DC: 0 xford University Press; 1997.

22. Lomnitz L. Supervivencia en una barriada de la Ciudad de México. En: Redes sociales, cultura y poder: ensayos de antropología latinoamericana. México, D F: Porrúa-Flacso; 1994: 47-97.

23. González de la Rocha M. La reciprocidad amenazada: un costo más de la pobreza urbana. En: Enríquez-Rosas, ed. Hogar, pobreza y bienestar en México. México, D F: Instituto Tecnológico y de Estudios Superiores de 0 ccidente (ITESO); 1999.

24. Enríquez R. El rostro actual de la pobreza urbana en México. Comercio Exterior 2003;53:532-539.

25. Dabas E. La intervención en red. En: Dabas E, ed. Red de redes. Las prácticas de intervención en redes sociales. Buenos Aires, Argentina:Paidós; 1998:15-32.

26. Bronfman M. Como se vive se muere. Familia, redes sociales y muerte infantil. México, DF: Centro Regional de Investigaciones Multidisciplinarias-Universidad N acional Autónoma de México; 2000. 